\title{
Estrogen deprivation triggers an immunosuppressive phenotype in breast cancer cells
}

Daniela Hühn ${ }^{1,4}$, Pablo Martí-Rodrigo ${ }^{1,4}$, Silvana Mouron², Catherine S. Hansel'1, Kirsten Tschapalda' ${ }^{1}$ Bartlomiej Porebski', Maria Häggblad'1, Louise Lidemalm¹, Miguel A. Quintela-Fandino², Jordi Carreras-Puigvert ${ }^{1, *}$ \& Oscar FernandezCapetillo1,3,*

${ }^{1}$ Science for Life Laboratory, Division of Genome Biology, Department of Medical Biochemistry and Biophysics, Karolinska Institute, S-171 21 Stockholm, Sweden

${ }^{2}$ Breast Cancer Clinical Research Unit, Spanish National Cancer Research Centre (CNIO), Madrid 28029, Spain

${ }^{3}$ Genomic Instability Group, Spanish National Cancer Research Centre (CNIO), Madrid 28029, Spain

${ }^{4}$ Co-first authors

${ }^{*}$ Co-corresponding authors 


\begin{abstract}
Estrogen receptor (ER)-positive breast tumors are routinely treated with estrogen-depriving therapies. Despite their effectiveness, patients often progress into a more aggressive form of the disease. Through a chemical screen oriented to identify chemicals capable of inducing the expression of the immune-checkpoint ligand PD-L1, we found antiestrogens as hits. Subsequent validations confirmed that estrogen deprivation or ER $\alpha$ depletion induces PD-L1 expression in ER-positive breast cancer cells, both in vitro and in vivo. Likewise, PD-L1 expression is increased in metastasis arising from breast cancer patients receiving adjuvant hormonal therapy for their local disease. Transcriptome analyses indicate that estrogen deprivation triggers a broad immunosuppressive program, not restricted to PD-L1. Accordingly, estrogen deprived MCF7 cells are resistant to T-cell mediated cell killing, in a manner that can be reverted by estradiol. Our study reveals that while antiestrogen therapies effectively limit tumor growth in ER-positive breast cancers, they also trigger a transcriptional program that favors immune evasion.
\end{abstract}




\section{INTRODUCTION}

Breast cancer $(\mathrm{BC})$ is the most frequent cancer in women worldwide and the second-cause of cancer-related mortality (Bray, Ferlay et al., 2018). Around 75\% of $B C$ cases are tumors that express Estrogen Receptor alpha $(E R \alpha)$ and are dependent on its transcriptional activity for survival. Accordingly, therapies directed to limit ER $\alpha$ signaling such as ER antagonists (e.g. tamoxifen) or ER downregulators (e.g. fulvestrant) constitute the first-line treatment for $E R^{+} B C$ (reviewed in (Siersbaek, Kumar et al., 2018)). Specifically, the current standard of care is a combination of the hormone therapy with cyclin-dependent kinase 4/6 (CDK4/6) inhibitors, even though, until very recently, clinical trials had failed to see a significant increase in overall survival with this combination (Im, Lu et al., 2019). In any case, and while antiestrogen therapy is certainly effective, around one fifth of the patients relapse into a metastatic stage for which there is no cure. Because of this, there is an intensive effort in testing the efficacy of new therapies, to be used alone or in combination with hormone therapy, for the treatment of $\mathrm{ER}^{+} \mathrm{BC}$. Not surprisingly, there is particular interest in exploring the potential of therapies targeting immune checkpoints such as those mediated by cytotoxic-Tlymphocyte-associated antigen 4 (CTLA-4) and programmed death-1 (PD-1) (Wei, Duffy et al., 2018), although initial evidence indicate that ER+ tumors are not very responsive due to low numbers of infiltrating lymphocytes and a low mutational burden (Esteva, Hubbard-Lucey et al., 2019).

Estrogens, most frequently $17 \beta$-estradiol (E2), have widespread effects on transcription which are to a large extent mediated by binding to two members of the nuclear receptor family, ER $\alpha(E S R 1)$ and $\operatorname{ER} \beta$ (ESR2). Upon binding to estrogens, these factors homodimerize and bind to target sequences on chromatin where they regulate transcription preferentially at distal enhancers (Hah, Murakami et al., 2013, Li, Notani et al., 2013). Besides their well-known roles in reproductive organs, estrogens have generalized effects in other tissues including bone, liver, colon, adipose tissue, kidney, skin and the cardiovascular 
and central nervous systems (Eyster, 2016). In addition, emerging evidence indicates a particularly important role of estrogens in suppressing inflammation, which helps to explain the gender-related differences found in diseases such as multiple sclerosis or rheumatoid arthritis that are alleviated during pregnancy when estrogen levels peak (Confavreux, Hutchinson et al., 1998, Nelson \& Ostensen, 1997). On the contrary, the use of oral contraceptives reducing sex hormone levels has been shown to aggravate inflammatory bowel disease (Khalili, Higuchi et al., 2013). This immunosuppressive role of estrogens also plays an important role in cancers associated to inflammation such as hepatocellular carcinoma, which is three to five times more frequent in men than in women (Naugler, Sakurai et al., 2007). However, the contribution of this phenomenon to $\mathrm{BC}$ onset and response to hormone-therapy is still poorly understood. Here, by screening for molecules that modulate PD-L1 expression, we reveal a role for estrogens in suppressing a broad inflammatory transcriptional program in $\mathrm{ER}^{+} \mathrm{BC}$ cells, which limits their clearance by the immune system. 


\section{RESULTS}

\section{A chemical screen to identify regulators of PD-L1 expression}

In order to identify chemicals capable of regulating surface PD-L1 expression, we conducted a High-Throughput screen using 4,216 pharmacologically active compounds (Fig. 1A; see Methods for details). The screening was conducted in the human lung cancer cell line A549, which was previously shown to express PD-L1 upon interferon gamma (IFN $\gamma$ ) stimulation (Stanciu, Bellettato et al., 2006). Since we were originally more focused on discovering downregulators, the screening was conducted on A549 cells that were previously treated with 100 $\mathrm{ng} / \mathrm{ml}$ of IFN $\gamma$ for $24 \mathrm{hrs}$ and then subsequently with the compounds from the library for another $24 \mathrm{hrs}$. At this point, cells were stained with anti-PD-L1 antibodies, fixed and processed for High-Throughput Microscopy (HTM). As expected, wells treated with only IFN $\gamma$ (positive control) showed a significant increase of PD-L1 expression when compared with DMSO-treated negative controls. In this experimental setup, corticosteroids were the most enriched compound class found among the downregulators (Fig. 1B and Table S1). Subsequent validation experiments confirmed that 3 independent corticosteroids (dexamethasone, hydrocortisone and prednisone) significantly reduced the surface levels of PD-L1 in IFN $\gamma$-treated A549 cells (Fig. S1). Besides corticosteroids, inhibitors of the Janus kinases (JAK1/2) were also found among the downregulators, which are known to be involved in the IFN $\gamma$-dependent induction of PD-L1 (Hao, Chapuy et al., 2014). Noteworthy, this work can help to understand recent reports that either JAK1/2 mutations (Shin, Zaretsky et al., 2017) or baseline corticosteroid treatment (Arbour, Mezquita et al., 2018, Gourd, 2018) confer resistance to anti-PD-L1 therapy.

With regard to up-regulators, there were very few molecules capable of inducing PD-L1 beyond the levels observed with IFN $\gamma$, which we failed to confirm in subsequent validation experiments (in many cases the hits were related to autofluorescence of the compounds). Nevertheless, we were intrigued by the 
presence of the selective estrogen receptor degrader (SERD) fulvestrant among this list of hits, even if the screening was done in A549 cells, given that previous reports have indicated reduced lung cancer mortality risk in BC patients treated with antiestrogens (Bouchardy, Benhamou et al., 2011), and that fulvestrant has actually been shown to reduce the growth of A549 xenografts (Marquez-Garban, Chen et al., 2007). In order to further investigate the impact of anti-estrogen therapies on PD-L1 expression we switched to MCF7, which is a widely used $\mathrm{ER}+\mathrm{BC}$ cell line. First, in order to evaluate the effect of a complete hormone deprivation, we grew MCF7 cells in steroid-free media (SFM) for two weeks. This led to a clear upregulation of PD-L1, which as expected was present on the cell membrane (Fig. 1C). Using this experimental setup, we conducted a secondary chemical screen, where we tested the effects of 25 ER agonists and 11 ER antagonists in a dose-response (Fig. 1D, Table S2). Overall, ER agonists reduced and antagonists further increased PD-L1 expression in SFM-grown MCF7 cells.

\section{ER $\alpha$ signaling suppresses PD-L1 expression in ER ${ }^{+} B C$ cells}

To further evaluate the effect of estrogen signaling in $\mathrm{ER}^{+} \mathrm{BC}$ cells, MCF7 cells were either hormone-deprived in SFM or treated with fulvestrant for 2 weeks. While both conditions led to a clear upregulation of surface PD-L1 levels as detected by flow cytometry, only the effect of the SFM-treatment could be reverted with ethinylestradiol (EE) (Fig. 2A-D). Equivalent results were observed by microscopy (Fig. 2E). The lack of effect of EE in downregulating PD-L1 expression in fulvestrant-treated MCF7 cells suggests that the observed effects are due to $E R \alpha$, as the compound leads to the degradation of this receptor. Consistently, western blot analyses confirmed that EE reverted the increase in PD-L1 expression in SFM- but not fulvestrant-treated MCF7 cells, as the later lacked ER $\alpha$ expression (Fig. 2F). In addition, while SFM- or fulvestrant treatment induced PD-L1 expression in ER $\alpha^{+}$cells, it failed to do so in ER $\alpha^{-}$ones (Fig. S2). Quantitative reverse transcription polymerase chain reaction (qRT-PCR) 
analyses revealed that the upregulation of PD-L1 (CD274) levels in SFM- or fulvestrant-treated MCF7 cells occurred at the level of transcription (Fig. 2G,H). Finally, RNA interference-mediated downregulation of ER $\alpha$ (ESR1) but not ER $\beta$ (ESR2) also led to the upregulation of surface PD-L1 levels in MCF7 cells (Fig. S3). Collectively, these experiments reveal that estrogens suppress PD-L1 expression through the stimulation of ER $\alpha$ signaling.

\section{Estrogen deprivation triggers an inflammatory transcriptional program in MCF7 cells}

To determine the mechanism by which estrogen suppresses PD-L1 expression in $\mathrm{ER}^{+} \mathrm{BC}$ cells we analyzed how estrogen deprivation impacts the activation of Janus Kinase-Signal Transducer and Activator of Transcription proteins (JAKSTAT) and NF- $\mathrm{B} B$ signaling pathways, both of which are known regulators of PDL1 expression and play important roles in the sensitivity to cancer immunotherapies (Manguso, Pope et al., 2017, Pan, Kobayashi et al., 2018, Peng, Hamanishi et al., 2015, Shin et al., 2017). In fact, a time-course of MCF7 cells grown in SFM revealed that both pathways were activated, as evidenced by the phosphorylation of STAT1 at Tyr 701 (p-STAT1 ${ }^{\text {Tyr701) }}$ and RelA (p65) at Ser 536 ( $p-p 65^{\text {Ser536) }}$, concomitantly to the upregulation of PD-L1 (Fig. 3A). Interestingly, addition of EE after 16 days in SFM for the last 4 days reverted STAT1 but not p65 phosphorylation, arguing that the JAK/STAT pathway is the primary mediator of the observed effects. Nevertheless, both the JAK2 inhibitor (CEP-33779) or the NF-KB inhibitor (CAPE) reduced the upregulation of PD-L1 induced by SFM in MCF7 cells (Fig. 3B).

As to how JAK/STAT and NF- $\kappa \mathrm{B}$ signaling are activated upon estrogen deprivation, we found increased levels of IFN $\gamma$ and Tumor Necrosis Factor alpha (TNF $\alpha$ ) in SFM-grown MCF7 cells, which are the primary cytokines involved in the activation of each pathway, respectively (Fig. 3C, D). Interestingly, and besides IFN $\gamma$, estrogen deprivation also induced the secretion of IL-6, which is a 
central inflammatory cytokine that stimulates JAK/STAT signaling and that is known to decrease the effectiveness of cancer immunotherapy (Fig. 3E) (Johnson, O'Keefe et al., 2018). Of note, given that estrogen deprivation arrests the growth of MCF7 cells and that IL-6 is also an important component of the senescence associated secretory phenotype (SASP) (Faget, Ren et al., 2019), we wondered whether PD-L1 expression was part of a SASP response in these cells. However, even if MCF7 cells grown in SFM showed several features of senescence such as increased levels of $\mathrm{p} 21^{\mathrm{Cip} 1}$ and histone $\mathrm{H} 3$ lysine 9 trimethylation (H3K9me3) or an increased activity of the senescence-associated beta galactosidase (SA- $\beta$ gal) (Fig. S4A,B), MCF7 cells induced to undergo senescence upon treatment with the p53 activator Nutlin-3 failed to upregulate PD-L1 (Fig. S4C).

To obtain a general view of the transcriptional changes induced by estrogen deprivation in ER ${ }^{+}$BC cells, we conducted RNA sequencing (RNAseq) in MCF7 cells grown in SFM or with fulvestrant for 3 weeks. Analysis of Gene Set Enrichment Analysis (GSEA) hallmarks showed a good correlation between the transcriptional changes induced by both conditions (Fig. 3F). One of the common hallmarks was the Epithelial-Mesenchymal Transition (EMT), which is consistent with the change in morphology that is observed with these treatments. Moreover, and in support to the previous data, "TNF $\alpha$ signaling via NF-кB", "IFN $\gamma$ response" or "Inflammatory response" were amongst the most significantly induced hallmarks (Fig, 3F-I and Fig. S5). Thus, estrogen deprivation triggers a broad inflammatory transcriptional program in MCF7 cells, which includes the activation of known activators of PD-L1 expression such as NF-kB and IFN $\gamma$.

\section{Estrogen deprivation limits T-cell mediated cell killing of MCF7 cells}

To evaluate the extent to which the transcriptional program triggered by estrogen deprivation in BC cells affected their sensitivity to immune cells, we conducted a T-cell mediated cell killing assay in MCF7 cells. To do so, MCF7 cells stably 
expressing mCherry fused to a nuclear localization sequence were co-cultured in the presence of activated primary T-cells and followed by live cell imaging for 3 days. Remarkably, MCF7 cells that were previously grown on SFM or with fulvestrant were significantly resistant to their killing by T-cells (Fig. S6A). In addition, EE was able to alleviate the effects of the SFM treatment and increased the elimination of MCF7 cells by T-cells. Equivalent results were obtained by measuring apoptosis through the use of a fluorescent caspase-3/7 target (Fig. S6B). As expected, no cell killing was observed when MCF7 cells were cocultured in the presence of T-cells that were not previously activated (Fig. S6C,D). Thus, estrogen deprivation increases the resistance of ER+MCF7 cells to their killing by $\mathrm{T}$ lymphocytes.

\section{ER $\alpha$ inversely correlates with PD-L1 expression in breast cancer}

Recent analyses of The Cancer Genome Atlas (TCGA) project have indicated higher levels of PD-L1 in Triple Negative Breast Cancer (TNBC) when compared to non-TNBC subtypes (Mittendorf, Philips et al., 2014). Based on our findings, we explored whether this correlation could be linked to the expression of ER $\alpha$. Indeed, gene expression analysis of $1904 \mathrm{BCs}\left(E R \alpha^{+}: 1459\right.$; $\left.E R \alpha^{-}: 445\right)$ from the METABRIC cohort of the TCGA dataset revealed significantly higher levels of PD-L1 mRNA expression (CD274) in the ER $\alpha^{-}$cohort (Fig. 4A). Similar results could be observed at the protein level when comparing PD-L1 expression between 5 ER $\alpha^{+}$cell lines (MCF-7, T47D, CAMA-1, ZR-75-1 and BT-474) and 3 ER $\alpha^{-}$ones (MDA-MB-231, HCC1937 and BT-549) (Fig. 4B). Next, to determine if PD-L1 expression was also induced in vivo in response to an anti-estrogen treatment we used a transgenic mouse model of $E R^{+} B C$ based on the expression of the Polyoma Virus middle $\mathrm{T}$ Antigen downstream of the mouse mammary tumor virus long terminal repeat (MMTV-PyMT) (Guy, Cardiff et al., 1992). In agreement with our in vitro findings, treatment of MMTV-PyMT transgenic mice harboring $\mathrm{BC}$ with the ER agonist tamoxifen led to a significant increase in PDL1 expression in the tumors (Fig. 4C,D). Finally, we evaluated PD-L1 expression 
in tissue from a small cohort of human patients of $\mathrm{ER}^{+} \mathrm{BC}$ (clinical and demographic characteristics detailed on Table S3) for which we obtained paired biopsies from the primary tumor and the metastases that emerged during or after adjuvant hormonal therapy. While PD-L1 expression was virtually absent in the primary tumors, areas of PD-L1 expressing cells could be detected in half of the metastatic samples (Fig. 4E,F).

\section{DISCUSSION}

Estrogen was one of the first hormones to be described and for a while was thought to only act in the female reproductive system (Eyster, 2016). Later studies revealed that estrogen receptors were widely expressed in many organs, and that estrogens played pleotropic physiological roles beyond reproduction. Among these, several studies have indicated that estrogen levels influence the severity of diseases linked to inflammation, including cancer (Khalili et al., 2013, Naugler et al., 2007, Nelson \& Ostensen, 1997). However, somewhat surprisingly, the relevance of the link between estrogen signaling and inflammation has not been particularly focused on $\mathrm{ER}^{+} \mathrm{BC}$, which is the cancer type where estrogen signaling plays a central role. As to how ER modulates inflammation, prior evidence had indicated a suppressive role of ER $\alpha$ in NFKB (Kalaitzidis \& Gilmore, 2005). Consistent with our findings, a recent study also indicated a role for $E R \alpha$ in regulating PD-L1 transcription, although the mechanism was unknown (Liu, Shen et al., 2018). Here, we show that the impact of estrogen deprivation is not restricted to a specific effect of PD-L1 but rather triggers a broad inflammatory transcriptional program in $\mathrm{ER}^{+} \mathrm{BC}$ cells, which includes the secretion of cytokines that activate $\mathrm{NF}_{\kappa \mathrm{B}}$ signaling such as $\mathrm{TNF} \alpha$, but also additional cytokines such as IFN $\gamma$ and IL-6 that trigger the activation of the JAK/STAT pathway. The immunosuppressive phenotype of estrogen-deprived $E R^{+} B C$ cells includes the expression of immune checkpoints such as PD-L1, which limit their clearance by the immune system. This phenotype switch that occurs upon estrogen deprivation should be considered when considering cancer immunotherapy 
regimens for the treatment of $\mathrm{ER}^{+} \mathrm{BC}$. Of note, and besides estrogen signaling, our chemical screen also identified corticosteroids as the most prominent class of drugs that reduces IFN $\gamma$-induced PD-L1 expression, providing an explanation for recent observations that indicate a reduced efficiency of anti-PD1/PD-L1 immunotherapies in patients undergoing a baseline treatment with corticosteroids (Gourd, 2018).

$\mathrm{BC}$ has classically been considered as poorly responsive to immunotherapy due to initial failures in vaccination or cytokine treatments in the 1980's and 90's (Buzdar, Blumenschein et al., 1984, Miles, Roche et al., 2011), and due to the fact that $\mathrm{ER}^{+} \mathrm{BC}$ has a low mutational burden and is therefore immunologically "cold". Nevertheless, metastatic BC is still the second cause of cancer-related mortality worldwide and new therapies are urgently needed. In this context, and due to the impressive effects that have been seen in patients of cancers with dismal prognosis such as melanoma or lung cancer, the efficacy of cancer immunotherapy is now a very active area of clinical investigation in the treatment of BC. As of September 2018, there were 285 clinical trials related to immunotherapy in BC, 208 of which were related to targeting the PD-1/PD-L1 checkpoint (reviewed in (Esteva et al., 2019)). However, since hormone therapy is a safe and effective approach for the initial treatment of $E R^{+} B C$, clinical trials are mostly oriented to either TNBC or to metastatic disease of any subtype. Still, around one fifth of the $\mathrm{ER}^{+} \mathrm{BC}$ patients undergoing hormone therapy progress into metastasis. Based on our work, we suggest that a combination of hormone therapy with therapies targeting PD-1 or PD-L1 for the treatment of primary ER+ $\mathrm{BC}$ might facilitate the clearance of the residual $\mathrm{BC}$ cells that persist even at low growth rates, and thus reduce the percentage of patients that progress into metastatic disease. 


\section{METHODS}

\section{Cell culture, transfection and chemicals}

A549, MCF7, T47D, HCC1937 and MDA-MB-231 cell line identity was confirmed using short-tandem repeat profiling analysis by ATCC. Except HCC1937, which were cultured in RPMI-1640, all cell lines were cultured in Dulbecco's modified Eagle's medium supplemented with $10 \%$ fetal calf serum and penicillin/streptomycin $(100 \mathrm{U} / \mathrm{ml})$ at $37^{\circ} \mathrm{C}$ in a $5 \% \mathrm{CO}_{2}$ humidified incubator.

In experiments requiring hormone depletion, cells were cultured in phenol redfree DMEM supplemented with $2 \mathrm{mM}$ L-glutamine and $10 \%$ charcoal-dextran stripped fetal calf serum (Sigma-Aldrich, F6765), hereafter termed steroid-free media (SFM). Where indicated 10nM 17a-Ethynylestradiol (Sigma-Aldrich, E4876) was added to SFM. For fulvestrant treatments, cells were cultured in standard DMEM supplemented with $1 \mu \mathrm{M}$ fulvestrant (ICI 182,780, Tocris, 1047) for the indicated number of days. During all treatments the respective media was exchanged every 4 days. For siRNA transfections, cells were seeded in 6-well plates and transfected the next day with 30pmol of ESR1 (Sigma-Aldrich, SASI_Hs01_00078592), ESR2 (Dharmacon, L-003402-00-0005) or Ctrl (Dharmacon, D-001810-10-20) siRNA using RNAimax according to the manufacturer's instructions. Transfection was repeated 3 days later and cells were harvested for analysis at day 6.

\section{High-throughput Screening (HTS)}

The chemical compound library was provided by the Chemical Biology Consortium Sweden (CBCS) and contained 4,126 pharmacologically active compounds from the following libraries: Prestwick, Tocris mini, Selleck tool compounds, Selleck known kinase inhibitors and ENZO tool compounds as well as 115 covalent drugs synthesized by Henriksson M. (Karolinska Institutet, Sweden). Plate and liquid handling were performed using Echo550 (Labcyte), Viaflo 384 (Integra Bioscience) and Multiflo FX Multi-Mode Dispenser (BioTek). Images were acquired by IN Cell Analyzer 2200 (GE Healthcare) with a 4x 
objective and quantitative image analyses were run in CellProfiler (www.cellprofiler.org) (Jones, Kang et al., 2008). Statistical analyses were carried out with Microsoft Excel and Graphpad Prism softwares. For the primary HTS screening, A549 cells were trypsinized, resuspended in culture medium containing 100ng/ml human IFN $\gamma$ (Sigma-Aldrich), dispensed into 384-well plates (BD Falcon, 353962) and incubated for $24 \mathrm{~h}$ at $37^{\circ} \mathrm{C}$ in a $5 \% \mathrm{CO}_{2}$ atmosphere. The next day, compounds were added to cells achieving a final concentration of $10 \mu \mathrm{M}$ and a DMSO volume concentration of $0.1 \%$. Cells were incubated for $24 \mathrm{~h}$ before staining with PE-labeled anti-human CD274 (Clone MIH1, BD Biosciences) antibody. Cells were fixed and stained with $2 \%$ formaldehyde and 2 $\mu \mathrm{M}$ Hoechst 33342, respectively. For the validation screening, MCF-7 cells were hormone-stripped by pre-culturing in SFM for 15 days with several media changes. Cells were subsequently exposed to DMSO or ethinylestradiol (EE) for 3 days, seeded in 384 -well plates and incubated overnight at $37^{\circ} \mathrm{C}$ in a $5 \% \mathrm{CO}_{2}$ atmosphere. The next day, the chemical library, comprising 163 compounds, including estrogens and antiestrogens, was added to the cells reaching a final concentration of $0.1,1.0$ and $10 \mu \mathrm{M}$, respectively. After $72 \mathrm{~h}$ of incubation, cells were stained and fixed as described above.

\section{Immunofluorescence microscopy}

Cells were grown in 96-well imaging plates (BD Falcon, 353219) and directly stained with PE-labeled anti-human CD274 antibody (Clone MIH1, BD Biosciences) before washing with PBS and fixation with $2 \%$ formaldehyde. Subsequently, cells were permeabilized in $0.25 \%$ Triton X-100 in PBS and blocked in 3.5\% BSA-PBS. Cells were stained with anti-ER $\alpha$ antibody (CST, 8644) diluted in blocking solution. Nuclei were stained with $2 \mu \mathrm{M}$ Hoechst 33342. Images were acquired on a Nikon eclipse Ti2 inverted wide-field microscope using a 20x 0.75 NA objective. 


\section{Immunoblotting}

Cells were lysed in RIPA buffer (Thermo Fisher Scientific) supplemented with protease and phosphatase inhibitor cocktail (Roche), sonicated for 5 min and centrifuged at $4^{\circ} \mathrm{C}, 14000 \mathrm{rpm}$ for $15 \mathrm{~min}$. $50 \mu \mathrm{g}$ whole-cell extracts were separated by SDS-PAGE and transferred onto Nitrocellulose membrane (BioRad). After blocking in 5\% milk in TBST, immunodetection was done overnight at $4^{\circ} \mathrm{C}$ with antibodies against PD-L1 (CST, 13684), ER $\alpha$ (CST, 8644) Stat1 (CST, 14994), phospho-Stat1 ${ }^{\text {Tyr701 }}$ (CST, 9167), p65 (CST, 8242), phospho-p65 ${ }^{\text {Ser536 }}$ (CST, 3033), $\beta$-actin (Abcam, ab6276) and GAPDH (Millipore Sigma, ab2302). Appropriate HRP-coupled secondary antibodies diluted in blocking solution were incubated for $1 \mathrm{~h}$ at room temperature. Signals were visualized by chemiluminescence (SuperSignal ${ }^{\mathrm{TM}}$ West Dura, Thermo Scientific, 34076) and acquired by an Amersham Imager 600 (GE healthcare).

\section{Flow cytometry}

Cells were cultured as indicated and harvested using Accutase (BD Biosciences, 561527). After centrifugation, cells were stained with PE-labeled anti-human PDL1 antibody (Clone MIH1, BD Biosciences) diluted in 2\% FCS-PBS blocking solution for $45 \mathrm{~min}$ at $4^{\circ} \mathrm{C}$. Samples were washed and immediately measured on a Guava easyCyte flow cytometer (EMD Millipore). Data were analyzed with Guava InCyte and GraphPad Prism software.

\section{qRT-PCR}

Total RNA was isolated using the PureLink RNA mini kit (Invitrogen) according to the manufacturer's instructions. Reverse transcription and PCR amplification were performed using TaqMan RNA-to-CT 1-Step Kit and the StepOnePlus ${ }^{\text {TM }}$ real-time PCR instrument (Applied Biosystems). The following probes were used in this study: Hs01125301_m1 for CD274, Hs99999901_s1 for 18S, Hs03929097_g1 for GAPDH, Hs01046817_m1 for ESR1, Hs01100353_m1 for ESR2, Hs00174128_m1 for TNFA, Hs00989291_m1 for IFNG. 


\section{Cytokine analysis}

MCF-7 cells were cultured in different media for indicated number of days and supernatant culture media was collected every 4 days, centrifuged and stored at $-80^{\circ} \mathrm{C}$ until analysis. IL-6 levels of culture supernatants were determined using the LEGENDplex ${ }^{\text {TM }}$ Human Inflammation Panel I (BioLegend, 740809), according to the manufacturer's instructions. Briefly, supernatants $(50 \mu \mathrm{L}$ /sample) were incubated with capture beads for $2 \mathrm{~h}$ at room temperature on an orbital shaker. Next, detection antibody was added and beads were incubated for $1 \mathrm{~h}$ at room temperature. After washing the beads, samples were measured using a BD LSRFortessa ${ }^{\mathrm{TM}}$ flow cytometer (BD Biosciences). Cytokine concentration was calculated based on a standard curve using BioLegend's LEGENDplex ${ }^{\mathrm{TM}}$ data analysis software.

\section{Gene expression analysis}

Next-generation RNA sequencing was performed to determine changes in gene expression between DMEM (normal), SFM (Treatment 1), fulvestrant (Treatment 2) or SFM+EE (Treatment 3) cultured MCF-7 cells. Cells were cultured during 20 days in DMEM, SFM or fulvestrant, and 20 in SFM with addition of ethinylestradiol the last 4 days. The cells were subsequently harvested and frozen at $-80^{\circ} \mathrm{C}$, and the samples were processed by Eurofins Genomics Sweden AB, were the RNA was isolated and assessed for QC, and finally cDNA library preparation was performed. Illumina single read sequencing with a read length of $1 \times 50 \mathrm{bp}$ and 30million reads per sample was performed.

\section{RNA sequencing data analysis}

For gene set enrichment analysis (GSEA), genes in each condition were ranked based on the log2 (fold change) value between DMEM (normal) and SFM (Treatment 1) or fulvestrant (Treatment 2). Each treatment was done in triplicate. Genes enriched in each treatment were positive and genes enriched in normal 
conditions (DMEM) were negative. The ranked gene lists were loaded into GSEA software and tested against the gene sets of the Hallmark collection (MSigDB).

\section{Data availability}

RNA sequencing data associated to this work are accessible at the GEO repository, under accession number GSE134938.

\section{T-cell mediated tumor cell killing assay}

MCF7 cells were transfected with mCherry-Nucleus-7, a gift from Michael Davidson (Addgene plasmid \# 55110), and a clone was selected. This MCF7 clone was then subjected to SFM, SFM with 10nM ethinylestradiol and fulvestrant treatments, as described above. T-cells were isolated 5 days prior to addition to MCF7 cells and were activated with Dynabeads ${ }^{\circledR}$ Human T-Activator CD3/CD28 (Thermofisher, 11131D) and $2.5 \mathrm{ng} / \mathrm{mL}$ of recombinant human IL-2 (Thermofisher, PHC0027). T-cells were co-cultured with the mCherry-Nucleus-7 MCF7 cells in the presence of CellEvent ${ }^{\mathrm{TM}}$ Caspase-3/7 Green Detection Reagent (ThermoFisher, C10423) in 96 well plates. Images were captured every 2 hours on a MetaXpress Microscope (Molecular Devices). Total MCF7 nuclear count and caspase intensity was analysed using CellProfiler software.

\section{TCGA data set analysis}

mRNA expression levels of CD274 from the METABRIC breast cancer TCGA data set (Pereira, Chin et al., 2016) were retrieved from cBioportal. Only patients with tumor mRNA data were taken into consideration $(n=1904)$. The samples were then classified as $E R \alpha+$ or $E R \alpha$ - using the annotation included in the dataset. The expression levels present in cBioportal are automatically transformed into Z-scores for comparison purposes. Two-tailed Student's t-test was used to assess the statistically significant differences in CD274 mRNA expression levels between $\mathrm{ER} \alpha^{+}$or $\mathrm{ER} \alpha^{-}$patients. 


\section{Mouse study}

PyMT [FVB/N-Tg(MMTV-PyVT)634Mul/J] transgenic animals harboring breast tumors were treated with 4-hydroxytamoxifen (Sigma-Aldrich) (1.2 mg/kg/daily) in $10 \%$ ethanol in sunflower oil by oral gavage. Animals were killed in $\mathrm{CO}_{2}$ chamber when tumors reached the humane end point, and the tumors were fixed in $10 \%$ formalin solution and embedded in paraffin. For purification of total RNA from formalin-fixed tumor sections, RNeasy FFPE kit (Qiagen) were used following manufacturer instructions. Reverse transcription was done using SuperScriptTM IV VILOTM Master Mix (ThermoFisher Scientific) and the real time PCR was performed using Fast SYBRTM Green Master Mix (Applied Biosystems) in a 7500 Fast Real-Time PCR system (Applied Biosystems). The following primers were used for Cd274 (FW: 5' TGCGGACTACAAGCGAATCA and REV: 5' GCTGGATCCACGGAAATTC) and $\beta$-actin detection (FW: 5' GGCTCCTAGCACCATGAAGA and REV: 5’ CCACCGATCCACACAGAGTA).

\section{Human study and tissue}

Women with a histologic diagnosis of HRPBC, for whom tissue from a distant metastasis and full medical records were available, were eligible. Patients with synchronous metastases were excluded. The study protocol was approved by the Institutional Review Board of Hospital 12 de Octubre ("Comité Ético de Investigación Clínica - Hospital 12 de Octubre", Madrid, Spain) (Study code: 11/137), and conducted according to the principles expressed in the Declaration of Helsinki. This review board waived the need for consent since all the samples belonged to patients diagnosed of cancer before 2007. According to the Royal Act in Biomedical Research in force in Spain since 2007 (Royal Act 14/2007, July $\left.3^{\text {rd }}\right)$, the retrospective collection of archival samples belonging to patients diagnosed before 2007 do not require individual signed informed consent. 


\section{Immunohistochemistry}

For histological analyses, tissues were fixed in 10\% buffered formalin (SigmaAldrich; St. Louis, MO, USA) and embedded in paraffin. Immunohistochemical staining with anti-PD-L1 antibody (Rabbit monoclonal (E1L3N)- Cell Signaling \#13684) was performed on 2.5- $\mu$ m tissue sections. Immunohistochemistry was performed using an automated protocol developed for the Autostainer Link automated slide staining system (DAKO, Agilent). All steps were performed on this staining platform using validated reagents, including deparaffinization, antigen retrieval (cell conditioning), and antibody incubation and detection. Corresponding stainings were acquired and digitalized using the AxioScan.Z1 system (Zeiss). Digitalized images were automatically analyzed with the AxioVision version 4.6.2 software (Zeiss). The percentage of PD-L1 positivity was considered as ratio of PD-L1-positive cells to total number of cells.

\section{Statistics}

Statistical parameters and tests are reported in the Figures and corresponding Figure Legends. Statistical analysis was done using GraphPad Prism version 8.0 (GraphPad Software Inc). One-way-ANOVA was performed for all the datasets that required comparison among multiple data points within a given experimental condition. 


\section{ACKNOWLEDGEMENTS}

We would want to thank Andres J Lopez-Contreras for insightful comments on the manuscript. Research was funded by grants from the Cancerfonden foundation (CAN 2018/381) and the Swedish Research Council (VR) (538-201431) to OF and from the ISCIII (AES -PI16/00354; co-funded by the European Regional Development Fund) and from the Call for Coordinated Research Groups from Madrid Region, Madrid Regional Government-ERDF funds (B2017/BMD3733) to MQF.

\section{AUTHOR CONTRIBUTIONS}

D.H. and P.M-R. contributed to most experiments and data analyses and to the preparation of the figures. C.S.H. contributed to the T-cell killing experiments. S.M. and M.Q-F helped with experiments using MMTV-PyMT animals and with human patient material. M. H. helped with the chemical screen. L.L. provided general technical support to many of the experiments. O.F-C. and J.C-P. supervised the study and wrote the MS.

\section{DECLARATION OF INTERESTS}

The authors declare no competing interests. 


\section{REFERENCES}

Arbour KC, Mezquita L, Long N, Rizvi H, Auclin E, Ni A, Martinez-Bernal G, Ferrara R, Lai WV, Hendriks LEL, Sabari JK, Caramella C, Plodkowski AJ, Halpenny D, Chaft JE, Planchard D, Riely GJ, Besse B, Hellmann MD (2018) Impact of Baseline Steroids on Efficacy of Programmed Cell Death-1 and Programmed Death-Ligand 1 Blockade in Patients With Non-Small-Cell Lung Cancer. J Clin Oncol 36: 2872-2878

Bouchardy C, Benhamou S, Schaffar R, Verkooijen HM, Fioretta G, Schubert H, VinhHung V, Soria JC, Vlastos G, Rapiti E (2011) Lung cancer mortality risk among breast cancer patients treated with anti-estrogens. Cancer 117: 1288-95

Bray F, Ferlay J, Soerjomataram I, Siegel RL, Torre LA, Jemal A (2018) Global cancer statistics 2018: GLOBOCAN estimates of incidence and mortality worldwide for 36 cancers in 185 countries. CA Cancer J Clin 68: 394-424

Buzdar AU, Blumenschein GR, Smith TL, Powell KC, Hortobagyi GN, Yap HY, Schell FC, Barnes BC, Ames FC, Martin RG, et al. (1984) Adjuvant chemotherapy with fluorouracil, doxorubicin, and cyclophosphamide, with or without Bacillus CalmetteGuerin and with or without irradiation in operable breast cancer. A prospective randomized trial. Cancer 53: 384-9

Confavreux C, Hutchinson M, Hours MM, Cortinovis-Tourniaire P, Moreau T (1998) Rate of pregnancy-related relapse in multiple sclerosis. Pregnancy in Multiple Sclerosis Group. N Engl J Med 339: 285-91

Esteva FJ, Hubbard-Lucey VM, Tang J, Pusztai L (2019) Immunotherapy and targeted therapy combinations in metastatic breast cancer. Lancet Oncol 20: e175-e186

Eyster KM (2016) The Estrogen Receptors: An Overview from Different Perspectives. Methods Mol Biol 1366: 1-10

Faget DV, Ren Q, Stewart SA (2019) Unmasking senescence: context-dependent effects of SASP in cancer. Nat Rev Cancer

Gourd E (2018) Baseline corticosteroids reduce activity of PD-L1 blockade. Lancet Oncol 19: e515

Guy CT, Cardiff RD, Muller WJ (1992) Induction of mammary tumors by expression of polyomavirus middle T oncogene: a transgenic mouse model for metastatic disease. $\mathrm{Mol}$ Cell Biol 12: 954-61

Hah N, Murakami S, Nagari A, Danko CG, Kraus WL (2013) Enhancer transcripts mark active estrogen receptor binding sites. Genome Res 23: 1210-23

Hao Y, Chapuy B, Monti S, Sun HH, Rodig SJ, Shipp MA (2014) Selective JAK2 inhibition specifically decreases Hodgkin lymphoma and mediastinal large B-cell lymphoma growth in vitro and in vivo. Clin Cancer Res 20: 2674-83

Im SA, Lu YS, Bardia A, Harbeck N, Colleoni M, Franke F, Chow L, Sohn J, Lee KS, Campos-Gomez S, Villanueva-Vazquez R, Jung KH, Chakravartty A, Hughes G, Gounaris I, Rodriguez-Lorenc K, Taran T, Hurvitz S, Tripathy D (2019) Overall Survival with Ribociclib plus Endocrine Therapy in Breast Cancer. $N$ Engl J Med

Johnson DE, O'Keefe RA, Grandis JR (2018) Targeting the IL-6/JAK/STAT3 signalling axis in cancer. Nat Rev Clin Oncol 15: 234-248

Jones TR, Kang IH, Wheeler DB, Lindquist RA, Papallo A, Sabatini DM, Golland P, Carpenter AE (2008) CellProfiler Analyst: data exploration and analysis software for complex image-based screens. BMC Bioinformatics 9: 482

Kalaitzidis D, Gilmore TD (2005) Transcription factor cross-talk: the estrogen receptor and NF-kappaB. Trends Endocrinol Metab 16: 46-52 
Khalili H, Higuchi LM, Ananthakrishnan AN, Richter JM, Feskanich D, Fuchs CS, Chan AT (2013) Oral contraceptives, reproductive factors and risk of inflammatory bowel disease. Gut 62: 1153-9

Li W, Notani D, Ma Q, Tanasa B, Nunez E, Chen AY, Merkurjev D, Zhang J, Ohgi K, Song X, Oh S, Kim HS, Glass CK, Rosenfeld MG (2013) Functional roles of enhancer RNAs for oestrogen-dependent transcriptional activation. Nature 498: 516-20

Liu L, Shen Y, Zhu X, Lv R, Li S, Zhang Z, Shi YG, Tan L (2018) ERalpha is a negative regulator of PD-L1 gene transcription in breast cancer. Biochem Biophys Res Commun 505: 157-161

Manguso RT, Pope HW, Zimmer MD, Brown FD, Yates KB, Miller BC, Collins NB, Bi K, LaFleur MW, Juneja VR, Weiss SA, Lo J, Fisher DE, Miao D, Van Allen E, Root DE, Sharpe AH, Doench JG, Haining WN (2017) In vivo CRISPR screening identifies Ptpn2 as a cancer immunotherapy target. Nature 547: 413-418

Marquez-Garban DC, Chen HW, Fishbein MC, Goodglick L, Pietras RJ (2007) Estrogen receptor signaling pathways in human non-small cell lung cancer. Steroids 72: 135-43

Miles D, Roche H, Martin M, Perren TJ, Cameron DA, Glaspy J, Dodwell D, Parker J, Mayordomo J, Tres A, Murray JL, Ibrahim NK, Theratope Study G (2011) Phase III multicenter clinical trial of the sialyl-TN (STn)-keyhole limpet hemocyanin (KLH) vaccine for metastatic breast cancer. Oncologist 16: 1092-100

Mittendorf EA, Philips AV, Meric-Bernstam F, Qiao N, Wu Y, Harrington S, Su X, Wang Y, Gonzalez-Angulo AM, Akcakanat A, Chawla A, Curran M, Hwu P, Sharma P, Litton JK, Molldrem JJ, Alatrash G (2014) PD-L1 expression in triple-negative breast cancer. Cancer Immunol Res 2: 361-70

Naugler WE, Sakurai T, Kim S, Maeda S, Kim K, Elsharkawy AM, Karin M (2007) Gender disparity in liver cancer due to sex differences in MyD88-dependent IL-6 production. Science 317: 121-4

Nelson JL, Ostensen M (1997) Pregnancy and rheumatoid arthritis. Rheum Dis Clin North Am 23: 195-212

Pan D, Kobayashi A, Jiang P, Ferrari de Andrade L, Tay RE, Luoma AM, Tsoucas D, Qiu X, Lim K, Rao P, Long HW, Yuan GC, Doench J, Brown M, Liu XS, Wucherpfennig KW (2018) A major chromatin regulator determines resistance of tumor cells to T cellmediated killing. Science 359: 770-775

Peng J, Hamanishi J, Matsumura N, Abiko K, Murat K, Baba T, Yamaguchi K, Horikawa N, Hosoe Y, Murphy SK, Konishi I, Mandai M (2015) Chemotherapy Induces Programmed Cell Death-Ligand 1 Overexpression via the Nuclear Factor-kappaB to Foster an Immunosuppressive Tumor Microenvironment in Ovarian Cancer. Cancer Res 75: $5034-45$

Pereira B, Chin SF, Rueda OM, Vollan HK, Provenzano E, Bardwell HA, Pugh M, Jones L, Russell R, Sammut SJ, Tsui DW, Liu B, Dawson SJ, Abraham J, Northen H, Peden JF, Mukherjee A, Turashvili G, Green AR, McKinney S et al. (2016) The somatic mutation profiles of 2,433 breast cancers refines their genomic and transcriptomic landscapes. Nat Commun 7: 11479

Shin DS, Zaretsky JM, Escuin-Ordinas H, Garcia-Diaz A, Hu-Lieskovan S, Kalbasi A, Grasso CS, Hugo W, Sandoval S, Torrejon DY, Palaskas N, Rodriguez GA, Parisi G, Azhdam A, Chmielowski B, Cherry G, Seja E, Berent-Maoz B, Shintaku IP, Le DT et al. (2017) Primary Resistance to PD-1 Blockade Mediated by JAK1/2 Mutations. Cancer Discov 7: 188-201 
Siersbaek R, Kumar S, Carroll JS (2018) Signaling pathways and steroid receptors modulating estrogen receptor alpha function in breast cancer. Genes Dev 32: 1141-1154 Stanciu LA, Bellettato CM, Laza-Stanca V, Coyle AJ, Papi A, Johnston SL (2006) Expression of programmed death-1 ligand (PD-L) 1, PD-L2, B7-H3, and inducible costimulator ligand on human respiratory tract epithelial cells and regulation by respiratory syncytial virus and type 1 and 2 cytokines. J Infect Dis 193: 404-12

Wei SC, Duffy CR, Allison JP (2018) Fundamental Mechanisms of Immune Checkpoint Blockade Therapy. Cancer Discov 8: 1069-1086 


\section{FIGURE LEGENDS}

Figure 1. Small molecule screen for modulators of PD-L1 expression A, Overview of the phenotypic screen workflow. Briefly, A549 cells were seeded in $100 \mathrm{ng} / \mathrm{ml}$ IFN $\gamma 24 \mathrm{hrs}$ before addition of 4216 compounds at $10 \mu \mathrm{M}$. After $24 \mathrm{hrs}$ of compound exposure, cells were stained with an anti-PD-L1 antibody conjugated to phycoerythrin and fixed with formaldehyde. Nuclei were stained with Hoechst and the immunofluorescences were analysed by high-throughput microscopy (HTM). B, Hit distribution of the screen described in A illustrating the enrichment of JAK inhibitors and corticosteroids among the compounds reducing PD-L1 signal in HTM. PD-L1 levels in wells only stimulating with IFN $\gamma$ or negative controls (DMSO) are also shown. C, Immunofluorescence of PD-L1 (red) in MCF7 cells grown in normal or steroid-free medium (SFM) for 15 days. DAPI (blue) was used to stain DNA. D, Scatterplot of PD-L1 intensity levels in SFMgrown MCF7 cells treated with $\mathrm{ER} \alpha$ agonists or antagonists, screened at three concentrations, $0.1,1.0$ and $10 \mu \mathrm{M}$.

Figure 2. Estrogen-dependent suppression of PD-L1 expression in ER+ BC cells A, Flow cytometry mediated assessment of surface PD-L1 levels in MCF7 cells grown DMEM, SFM or SFM plus EE for 14 days. EE (10 nM) was added for the last 3 days. B, Flow cytometry mediated assessment of surface PD-L1 levels in MCF7 cells grown DMEM or fulvestrant $(1 \mu \mathrm{M})$ for 14 days. EE (10 nM) was added for the last 3 days. C,D Quantification from the data shown in $\mathbf{A}(\mathbf{C})$ and $\mathbf{B}$ (D). E, Immunofluorescence illustrating ER $\alpha$ (green) and PD-L1 (red) levels in MCF-7 cells cultured in DMEM, SFM or fulvestrant $(1 \mu \mathrm{M})$ for 20 days. Where indicated, EE (10 nM) was supplemented during the last 4 days. Representative images for each condition are shown. F, Western blot illustrating the levels of PDL1 and ER $\alpha$ in MCF-7 cells grown in DMEM, SFM or DMEM containing $1 \mu \mathrm{M}$ fulvestrant (Fulv) for 14 days. Where indicated EE (10nM) was added for the final 3 days. GAPDH levels are shown for loading control. G, H qRT-PCR analysis of 
PD-L1 (CD274) expression in MCF-7 cells cultured in DMEM and SFM (G) or fulvestrant $(1 \mu \mathrm{M}, \mathbf{H})$ for 18 days. Where indicated, media was supplemented with EE (10 nM) for the last 3 days. 18S RNA served as an internal control. * $p<0,05$; ${ }^{* *} p<0,01$

Figure 3. Estrogen signaling suppresses an inflammatory phenotype in MCF7 cells A, Whole-cell lysates from MCF-7 cells cultured in SFM for the specified days were analyzed by western blotting using the indicated antibodies. Where indicated, 10nM EE was added at 16 days for the final 4 days. Total p65 and $\beta$-ACTIN served as loading controls. B, Flow cytometry mediated evaluation of PD-L1 membrane levels in MCF-7 cells grown in DMEM or SFM for 21 days, alone or in combination with the NFkB inhibitor CAPE $(10 \mu \mathrm{M})$ or JAK2 inhibitor CEP-33779 $(10 \mu)$ for the last 3 days. C,D qRT-PCR analysis of IFN $\gamma$ (IFNG) (C) or TNF $\alpha$ (TNFA) (D) mRNA levels in MCF-7 cells cultured as in (A). 18S rRNA was used as an internal control. ${ }^{* *} p<0,01 \mathrm{E}$, Levels of IL-6 in the supernatant of MCF7 cells cultured in DMEM or SFM for the specified days as analyzed by LegendPlex-FACS (see Methods). Where indicated EE (10 nM) was added at day 17 for the last 4 days. F, GSEA Hallmark gene sets ranked by normalised enrichment score (NES) comparing the transcriptional programs triggered by 3 week treatments with fulvestrant $(1 \mu \mathrm{M})$ or SFM in MCF7 cells, both normalised to DMEM. Selected hallmarks are indicated in red. G,H,I Pre-ranked GSEA on the genes from the hallmarks "TNFA signaling via NF-kB" (G), "IFNG response" (H) and "Inflammatory response" (I) obtained from RNAseq analysis comparing the transcriptome of MCF7 cells grown in DMEM or SFM for 3 weeks. The heatmap representation illustrates the overall upregulation of these pathways in estrogen-deprived MCF7 cells.

Figure 4. Inverse correlation between ER $\alpha$ and PD-L1 in BC A, CD274 mRNA expression levels in $E R \alpha^{+}(n=1459)$ and $E R \alpha^{-}(n=445)$ patient samples. Normalised Z-scores were extracted from the METABRIC breast cancer TCGA 
dataset (Pereira et al., 2016). A factor was added to both populations in order to set the ER $\alpha+$ group to 0 . Data are presented as mean +SEM and two-tailed Student's t-test was used to calculate the statistical significance, ${ }^{* \star *} p<0.001$. B, Western blot analysis of PD-L1 and ER $\alpha$ protein in a panel of ER $\alpha^{+}$and $E R \alpha^{-} B C$ cell lines. $\beta$-Actin served as a loading control. $\mathbf{C}$, Schematics of the experimental procedure in mice. MMTV-PyVT mice that spontaneously develop mammary tumors were treated daily from week 5 until with 4-hydroxytamoxifen (4-OHT) or vehicle. D, Cd274 expression in tumors isolated from MMTV-PyVT mice treated with 4-OHT or vehicle as determined by RT-qPCR. Data were normalized to $\beta$ Actin mRNA levels. Graph represents the mean \pm SD and two-tailed Student's ttest was used to determine statistical significance, ${ }^{* *} p<0.01$. E, Immunohistochemical staining of PD-L1 (brown) in paired samples of primary and metastatic human ER+BC from two different patients. Sections were stained with Hematoxylin-Eosin to aid in the pathological analysis. F, Quantification of the percentage of PD-L1 positive cells in paired primary tumor and metastasis samples from 8 patients of ER $\alpha$-positive BC. 
Hühn D et al. Fig. 1

A

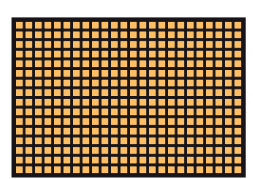

A549 cells seeding +/- IFN $\gamma$

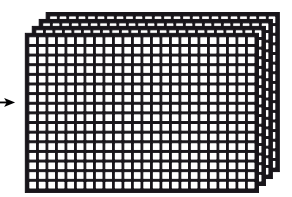

Compound adition

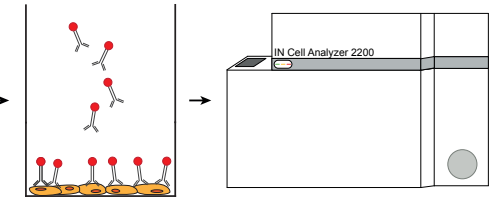

Live PD-L1 High-throughput staining
B

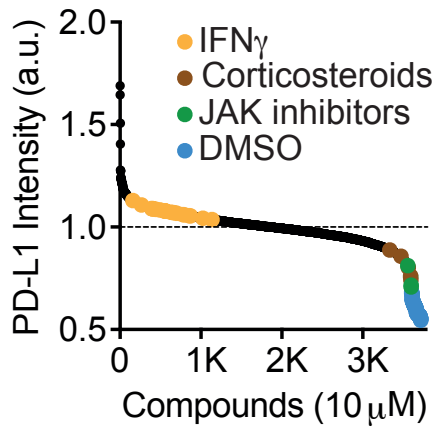

C

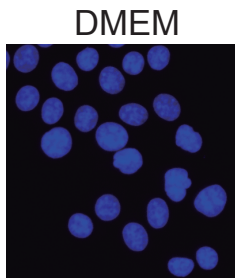

SFM

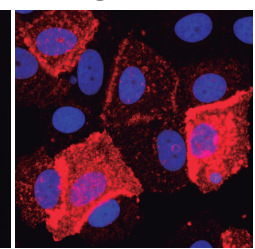

DAPI/PD-L1
D

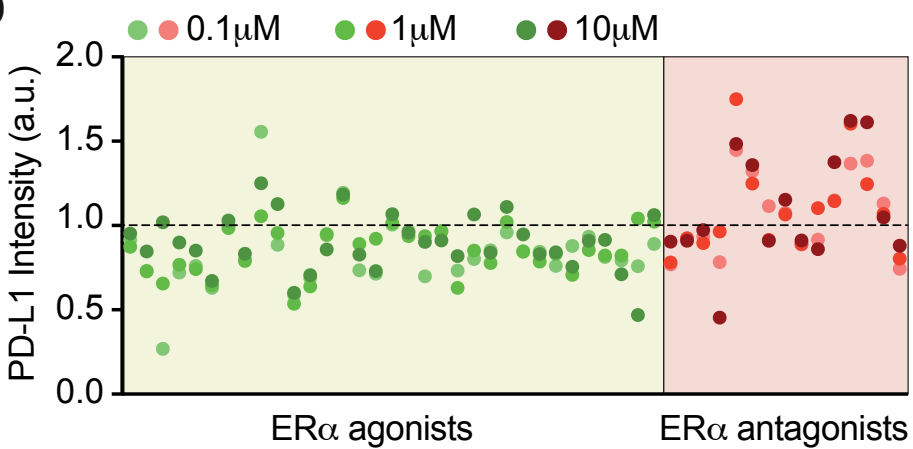


A B

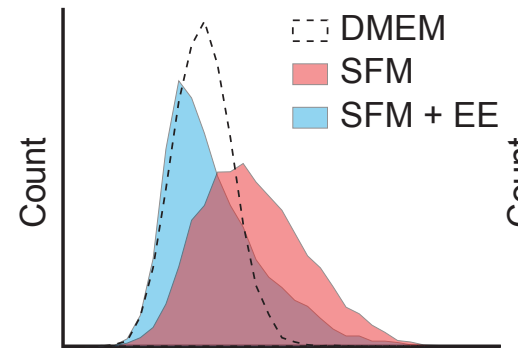

PD-L1

C

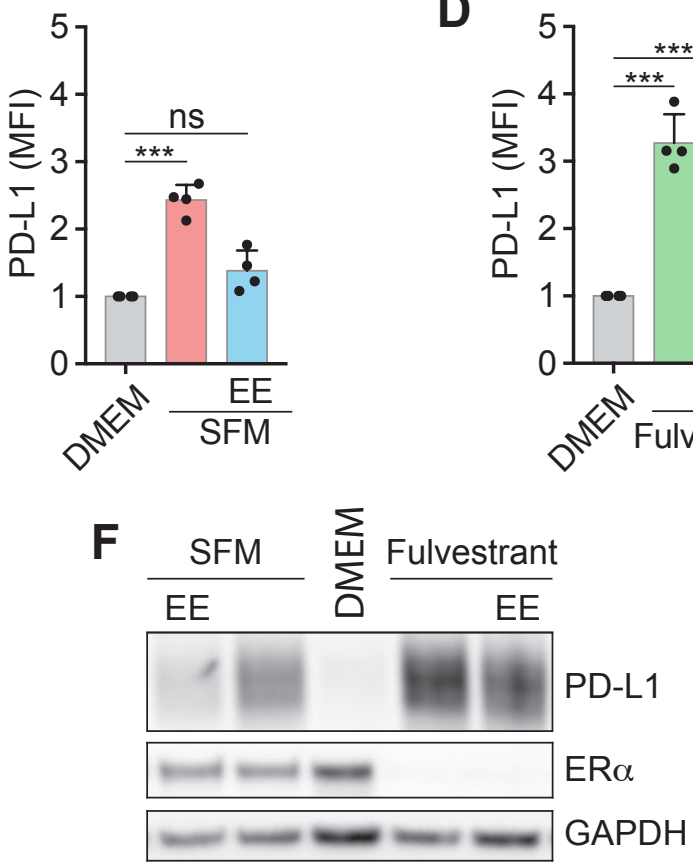

Hühn D et al. Fig. 2

E

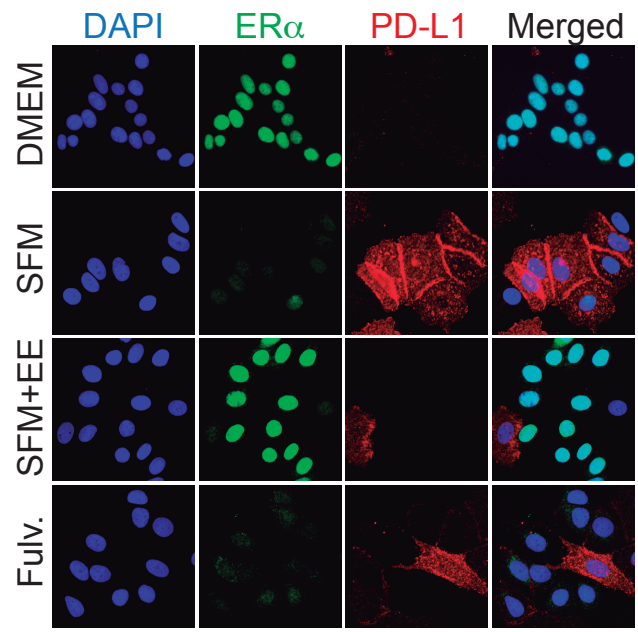

G

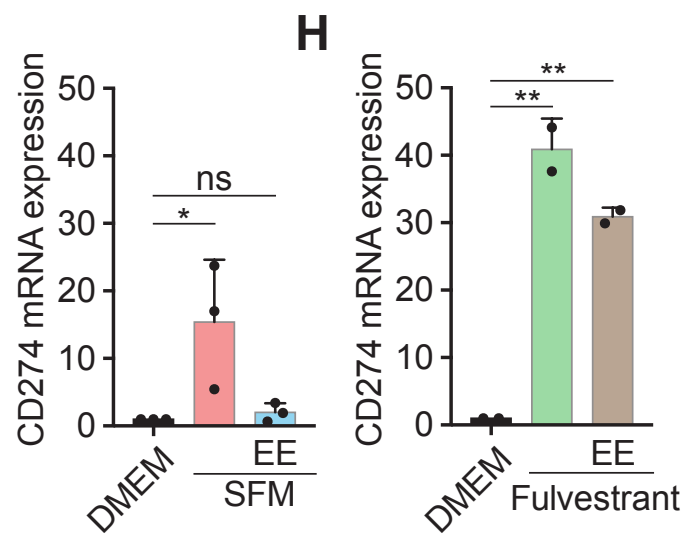


A

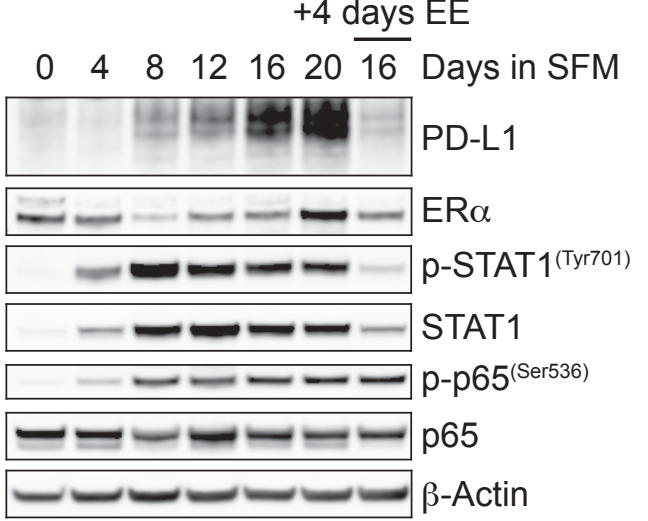

E

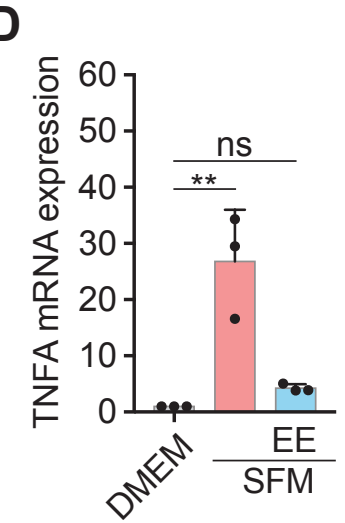

B

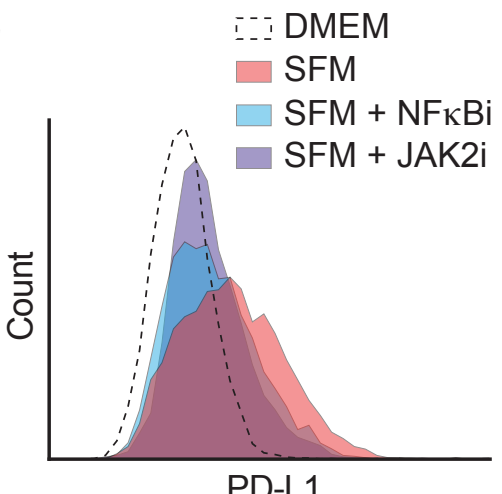

Hühn D et al. Fig. 3

C

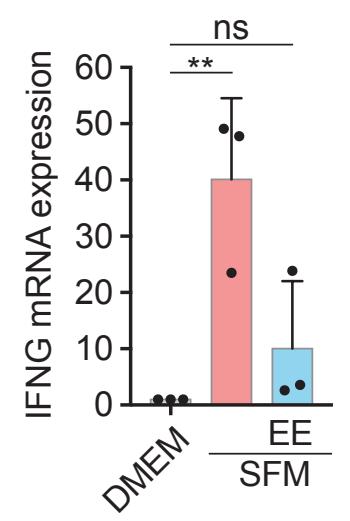

F

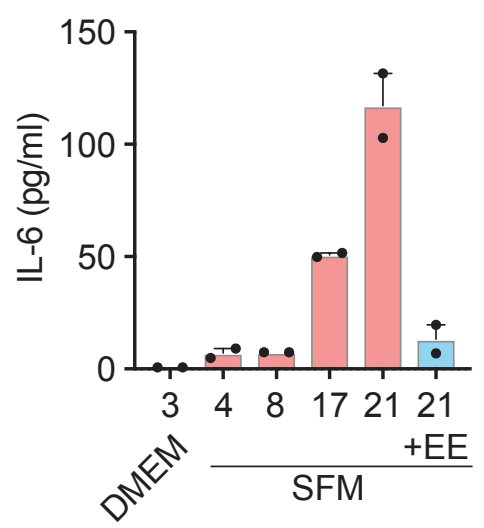

GSEA Hallmarks - NES

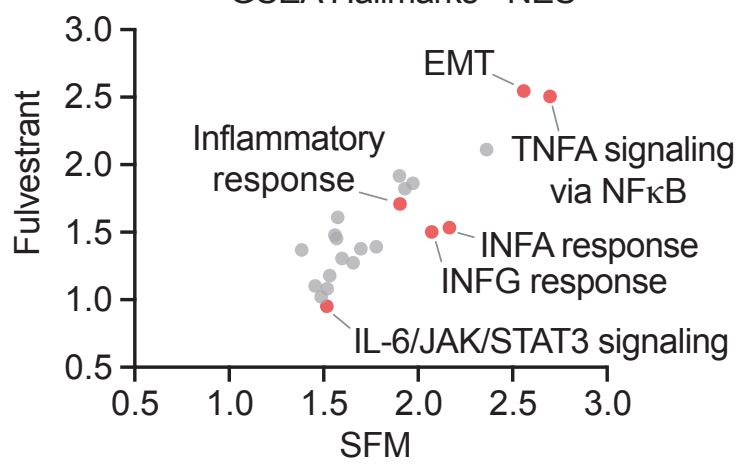

G

H

Ш

TNFA signaling via NFKB

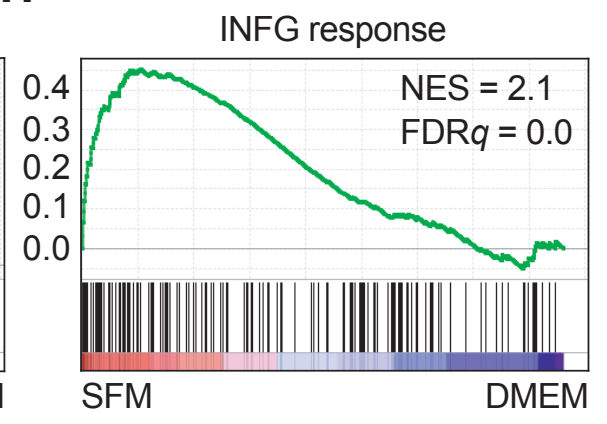

I

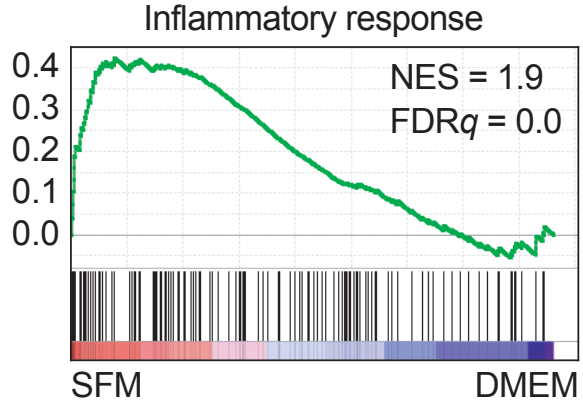

Gene rank in ordered dataset 
Hühn D et al. Fig. 4

A

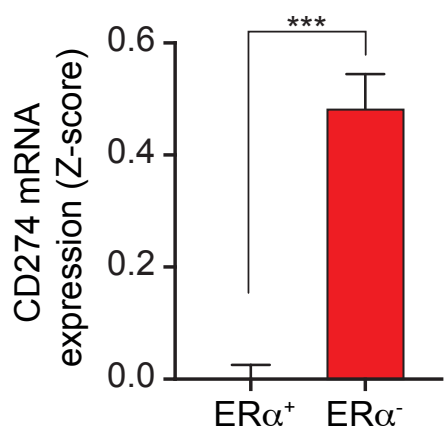

B
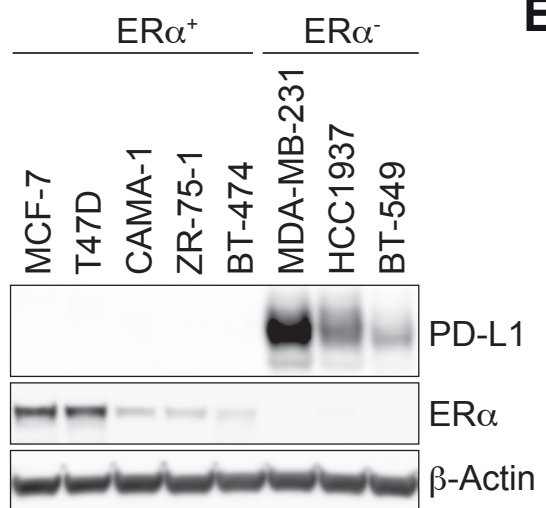

C

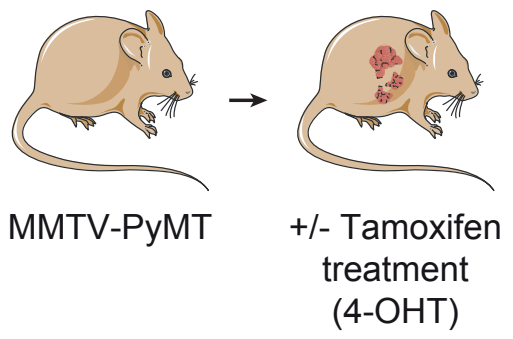

E

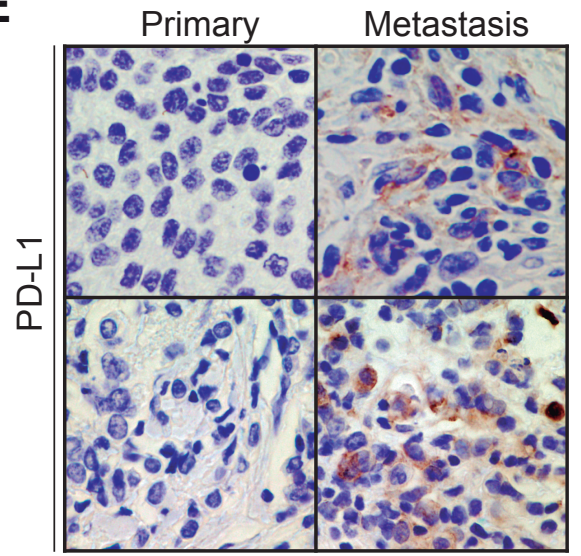

D

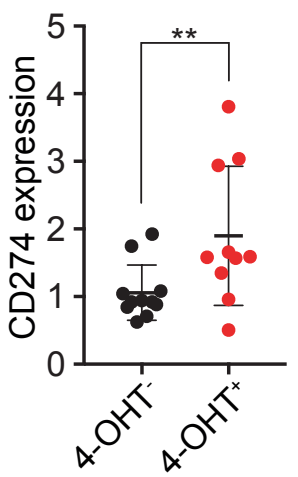

F

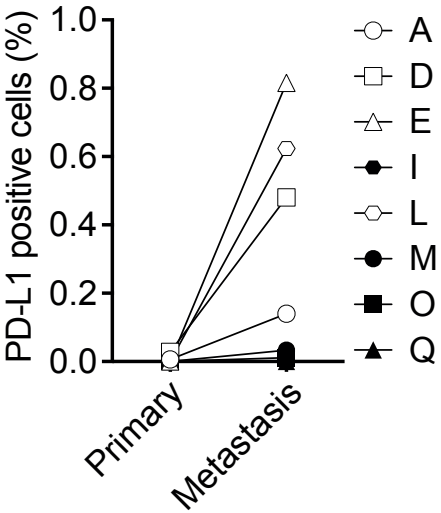

